\title{
Biofilms de Pseudomonas aeruginosa como mecanismos de resistencia y tolerancia a antibióticos. Revisión narrativa
}

\section{Biofilms of Pseudomonas aeruginosa as mechanisms of resistance and tolerance to antibiotics. Narrative review}

Andrés Felipe Bolívar-Vargas ${ }^{1}$, María Inés Torres-Caycedo ${ }^{1}$, Yaline Sánchez-Neira ${ }^{1}$

\section{RESUMEN}

La tolerancia antimicrobiana mediada por biofilms es un problema grave, principalmente en infecciones asociadas a la atención en salud. Esta mediada por diferentes mecanismos que expresa el biofilm como: la matriz de exopolisacaridos, alteraciones del microambiente, bacterias persistentes, seńal de quorum sensing, porinas, bombas de eflujo, expresión de genes, vesículas de membrana, ADN extracelular y enzimas. El objetivo de esta revisión fue identificar los mecanismos y efectos del

\begin{abstract}
Antimicrobial tolerance mediated by biofilms is a serious problem, mainly in infections associated with health care. It is mediated by different mechanisms expressed by the biofilm such as: the exopolysaccharide matrix, microenvironment alterations, persistent bacteria, quorum sensing signal, porins, efflux pumps, gene expression, membrane vesicles, extracellular DNA and enzymes. The objective of this review was to identify the mechanisms and effects of the Pseudomonas aeruginosa
\end{abstract}

\section{Historial del artículo:}

Fecha de recepción: 07/12/2020

Fecha de aprobación: 14/03/2021

1 Universidad de Boyacá, Facultad de Ciencias de la Salud, Bacteriología y laboratorio clínico, Tunja, Colombia

Correspondencia: Andrés Felipe Bolívar Vargas. Dirección: Carrera 2 Este No.64-169. Teléfono: 3042085434. Correo Electrónico: afbolivar@uniboyaca.edu.co

Como citar este artículo: Bolívar-Vargas AF, Torres-Caycedo MI, Sánchez-Neira Y. Biofilms de Pseudomonas aeruginosa como mecanismos de resistencia y tolerancia a antibióticos. Revisión narrativa. Revista de la Facultad de Ciencias de la Salud de la Universidad del Cauca. 2021;23(2):47-57. https://doi.org/10.47373/rfcs.2021.v232.1780 
biofilm de Pseudomonas aeruginosa en la resistencia a los antibióticos. Se realizó una revisión de la literatura sobre los principales mecanismos de tolerancia en antibióticos mediada por biofilms. Los biofilms aumentan la tolerancia de las bacterias gramnegativas a los diferentes tipos de antibióticos. Tras su exposición a cantidades minimas del mismo se genera la expresión de diferentes genes que expresan mecanismos que disminuyen la penetración y destrucción de los antibióticos. Aún no están bien definidos todos los factores que generan este tipo de tolerancia.

Palabras clave: Pseudomonas aeruginosa, Biofilm, Farmacorresistencia Bacteriana.

\section{INTRODUCCIÓN}

Pseudomonas aeruginosa es un patógeno ubicuo oportunista con forma de bastón (0.5-1 $\mu \mathrm{m}$ de diámetro y 1.5-5 $\mu \mathrm{m}$ de largo) que cuenta con un flagelo polar que le brinda motilidad (1). Son bacterias consideradas muy prevalentes en infecciones asociadas a la atención en salud (IAAS) ya que cuentan con una alta adaptación al cambio repetido del microambiente, disponibilidad de nutrientes, mecanismos de resistencia y capacidades para formar biofilms (2). Los biofilms son agregados bacterianos que se encuentran recubiertos por una matriz compuesta de exopolisacaridos, proteínas, enzimas y ácidos nucleicos, lo cual facilita su anclaje a superficies vivas e inertes como catéteres urinarios, marcapasos, tubos endotraqueales, catéteres endovenosos y prótesis valvulares $(2,3)$.

Se estima que el $65 \%$ de las infecciones bacterianas se debe a la formación del biofilm y a sus mecanismos de tolerancia a los antibióticos, lo cual representa un problema en el ámbito sanitario (2). Además, la formación de biofilms conduce a infecciones recurrentes, crónicas y al retardo en la cicatrización de las heridas $(3,4)$.

Los antibióticos utilizados para el tratamiento de infecciones causadas por este patógeno tienen como objetivos farmacológicos estructuras importantes de la bacteria como la pared celular, la síntesis de proteínas, el metabolismo bacteriano y los procesos de transcripción de ADN y ARN. Debido a la formación de biofilms y al uso prolongado de antibióticos, se presenta una adaptación y expresión de mecanismos de tolerancia por parte del microorganismo, lo cual conduce al fracaso terapéutico. Los mecanismos de resistencia mediados por ADN han cobrado una relevancia reciente dado que están implicados biofilm on antibiotic resistance. A literature review was carried out on the main mechanisms of tolerance in antibiotics mediated by biofilms. Biofilms increase the tolerance of gram-negative bacteria to different types of antibiotics. After exposure to minimal amounts of it, the expression of different genes that express mechanisms that decrease the penetration and destruction of antibiotics is generated. All the factors that generate this type of tolerance are not well defined yet.

Palabras clave: Pseudomonas aeruginosa, Biofilm, Farmacorresistencia Bacteriana.

en la resistencia del microrganismo en biofilms. La unión a plásmidos, principalmente en $P$. aeruginosa por su alto contenido de ácido desoxirribonucleico, puede generar a través del tiempo una adaptación evolutiva $(5,6)$. Si estos mecanismos se perpetúan, se puede esperar el desarrollo de cepas altamente resistentes.

El objetivo de la presente revisión narrativa es describir los mecanismos y efectos del biofilm de Pseudomonas aeruginosa en la resistencia y tolerancia a los antibióticos.

Se realizó una búsqueda amplia sobre los principales mecanismos de tolerancia a antibióticos producidos por los biofilms de Pseudomonas aeruginosa en bases de datos como Proquest, Science Direct, Scielo, Pubmed y Google Schoolar. Para ello, se utilizaron las palabras clave en inglés y espańol "Pseudomonas aeruginosa biofilms" AND "antibiotic tolerance"; "biofilms" AND "diseases"; "antimicrobial tolerance" AND "Biofilms", "infections associated with health care" AND "biofilm formation". Se localizaron un total de 70 artículos, de los cuales se tuvieron en cuenta estudios comprendidos entre los ańos 2015 al 2020, aquellos que incluyeran mecanismo de resistencia a partir de la formación de biofilms de Pseudomonas aeruginosa, infecciones frecuentes a causa de los biofilms de Pseudomonas aeruginosa y se excluyeron aquellos artículos que no contenían información acerca de los mecanismos de resistencia mediada por biofilms, infecciones bacterianas mediadas por biofilms en otras especies bacterianas patogenicidad de las infecciones mediadas por biofilms de otras especies. Finalmente se seleccionaron 52 artículos publicados en los últimos cinco ańos con los que se construyó una matriz que contiene el nombre del autor, ańo, título del artículo, resumen y conclusiones, con el fin de evaluar y comparar la información relevante. 


\section{INFECCIONES FRECUENTES CAUSADAS POR BIOFILMS DE PSEUDOMONAS AERUGINOSA}

Los biofilms de Pseudomonas aeruginosa, tienen una tolerancia extrema a los antibióticos en infecciones asociadas a atención en salud como neumonía. Adicionalmente, se asocian a enfermedades como fibrosis quística, heridas crónicas, otitis media, sinusitis crónica, infecciones músculo esqueléticas, infecciones del tracto urinario, endocarditis, prostatitis y queratitis; estas infecciones afectan a millones de personas cada ańo con una alta mortalidad asociada (7). En Europa se registran datos de resistencia asociada de $8.9 \%$ para infecciones causadas por Pseudomonas aeruginosa. Esto ha llevado a catalogar la resistencia como un problema epidemiológico. En Espańa, para el ańo 2017, fue reportado el 10.9\% de multirresistencias por este mismo microorganismo según el informe emitido por el "Centro Europeo para la Prevención y el Control de Enfermedades" (7). De la misma forma, en Estados Unidos uno de los principales agentes microbianos causantes de infecciones a nivel hospitalario es Pseudomonas aeruginosa, con reportes cercanos al 7.1\%; por su parte, en el ańo 2013 en México, el sistema de Vigilancia epidemiológica hospitalaria del Instituto Mexicano del Seguro Social registró una incidencia del $19.9 \%$.

En Colombia, el reporte de infecciones asociadas a la atención en salud (IAAS) durante los periodos de 2012 a 2017, documentó que, de 3932 aislamientos de bacilos gramnegativos, 1758 correspondían a Pseudomonas spp. Según el centro para el control y prevención de enfermedades de los Estados Unidos $(8,9)$, la Pseudomonas spp se presenta como "un patógeno de amenaza grave" y de acuerdo a la "Infectious diseases society of America" está catalogado dentro de los seis patógenos de máxima prioridad y resistencia a medicamentos (10-12).

La inserción de dispositivos médicos se ha vuelto indispensables para la medicina moderna, dentro de los cuales se encuentran: catéteres urinarios, catéteres vasculares, implantes ortopédicos, válvulas cardiacas y tubos endotraqueales (ETT). Estos dispositivos pueden recubrirse de una capa acondicionadora que pueden apoyar la adhesión bacteriana (13-15). Los ETT con frecuencia son implantados en pacientes con ventilación asistida y como consecuencia aumenta el riesgo de infecciones del tracto respiratorio superior, oclusión del dispositivo e infecciones de la herida. Una parte del biofilm se puede desprender y acceder a las vías respiratorias bajas a través del flujo de gas del ventilador, la aspiración o al momento de la inserción del ETT, con la subsecuente inoculación de bacterias orofaríngeas (16-18). Además, en los catéteres urinarios pueden migrar utilizando la superficie externa del catéter e introducirse directamente en la vejiga y gracias a los componentes acondicionadoras de la orina y proteínas del huésped generan un microambiente apropiado para su formación $(19,20)$.

\section{FORMACIÓN DEL BIOFILM}

La formación del biofilm se divide en cuatro etapas generales: adhesión, agregación, maduración y disgregación. En cuanto a la etapa de adhesión, las bacterias son atraídas al sustrato y se unen a la superficie por medio de diferentes tipos de fuerzas fisicoquímicas en las cuales se encuentra las fuerzas de Van Der Waals, fuerzas electrostáticas e hidrofóbicas (21). Algunas estructuras de estas bacterias como los flagelos y fimbrillas contribuyen a que esta unión sea más estable e irreversible, de esta forma les permite unirse a catéteres intravasculares, válvulas cardiacas o prótesis valvulares como se observa en la Figura 1.a (22).

Existen dos tipos de biofilms que generan las Pseudomonas aeruginosa, uno plano o inicial, el cual está caracterizado por presentar una confluencia uniforme de las bacterias en la superficie, y otro estructurado o maduro, el cual está compuesto por agregados bacterianos separados por canales (23-25).

Durante la etapa de agregación las células adheridas comienzan a dividirse y a producir matriz de exopolisacaridos (EPS) (Figura 1.b) (24). Posteriormente, el biofilm madura y hay una excesiva producción de proteínas, alginato, polisacáridos Pel, Psl (locis de genes que codifican EPS) y liberación del ADN extracelular. Este proceso le concede rigidez a la matriz de EPS, participa en la transferencia de genes de resistencia y el crecimiento del biofilm; además, en esta etapa la estructura tiene una arquitectura compleja con poros y canales los cuales trasportan desechos, agua y moléculas de quorum sensing (QS) (Figura1.c) (26).

Después, ocurre un aumento de manera exponencial de bacterias en el interior del biofilm y un aumento de los metabolitos, estos inducen la producción de enzimas (DNAasa, proteasas y fosfodiesterasa) que degradan la matriz extracelular y ayudan al desprendimiento de bacterias. Estas migran y forman grupos celulares que se adaptan a un nuevo entorno y colonizan a partir de la producción de sustancias de polímeros extracelulares (EPS), que permiten finalmente la formación del biofilm (Figura 1.d) (27).

La formación del biofilm está compuesta por 6 etapas. En la primera etapa (adhesión), las bacterias se unen por diferentes mecanismos al sustrato irreversiblemente, seguidamente ocurre la etapa de agregación en donde 
Figura 1. Esquema del proceso de formación del biofilm a partir de las etapas de a. adhesión, b. agregación, c. maduración, d. desprendimiento, e migración y f. colonización. Fuente: Autores.

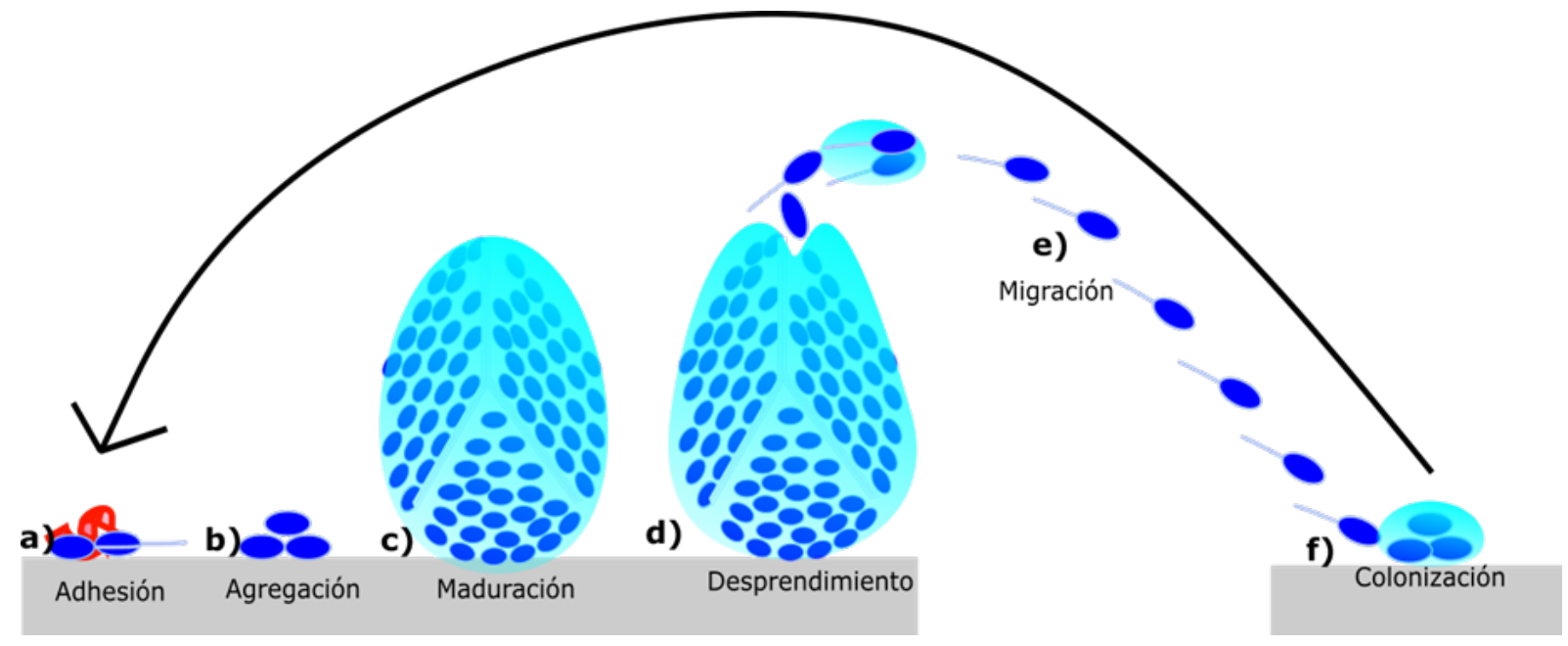

las bacterias unidas se replican y comienzan a producir la matriz de EPS. Después en la etapa de maduración el biofilm ya está completamente desarrollado con canales para para el transporte de agua, nutrientes y las seńales de quorum sensing; finalmente el biofilm comienza a desprenderse para migrar y colonizar otras superficies nuevas.

Dentro de las principales infecciones de origen bacteriano, se resalta aquellas causadas por bacterias formadoras de biofilm, las cuales representan alrededor de un $80 \%$ de las complicaciones en eventos de tipo crónico debido a los factores de virulencia y adaptación a diferentes entornos que hace que el impacto de las infecciones asociadas a la atención en salud se vea reflejado en aumentos de morbilidad y mortalidad a través de tiempo.

El proceso de regulación del Biofilm es complejo e implica varios sistemas de seńalización entrelazados, un componente importante en la regulación es el C-di-GMP el cual está involucrado en el cambio móvil a sésil de la Pseudomonas aeruginosa. Además, está involucrada la producción de polisacáridos dentro de ellos se encuentra el polímero de alginato formado por "ácido gulurónico" y "ácido manurónico", que confiere dentro del proceso de formación del biofilm la retención no solo de agua si no de nutrientes y estabilidad. Por otro lado la biosíntesis de los polisacáridos Psl yPel quienes intervienen en las primeras etapas de formación del biofilm $(11,26)$.

Otro sistema de regulación es la '5-difosfato-' 3-difosfato (ppGpp), la cual es regulada por las enzimas sintetasa monofuncional RelA y la sintetasa hidrolasa bifuncional Spot. En ciertas condiciones de estrés como la inanición de carbono, fosfato, aminoácidos, pH extremo y falta de osmolaridad, se inicia el proceso de síntesis de ppGpp el cual activa o inhibe la transcripción de genes para poder superar estas condiciones (28).

\section{FACTORES QUE FAVORECEN LA TOLERANCIA ANTIMICROBIANA}

\section{Matriz de exopolisacaridos (EPS)}

La matriz de EPS es uno de los componentes más complejos del biofilm y gracias a su composición y arquitectura le confiere cierta tolerancia al evitar la penetración del antibiótico al interior de la matriz extracelular (Figura 2.a) (29).

Los EPS funcionan como una barrera física la cual está conformada por moléculas aniónicas y catiónicas como glicoproteínas, glucolípidos y proteínas que impiden o retardan la absorción, transporte de antibióticos y acumulan hasta un $25 \%$ de su peso dentro de la matriz. Alrededor del $80 \%$ de su estructura se debe a la incorporación de agua lo que hace siempre este hidratada (30). Debido a la disminución de la absorción, el antibiótico llega en cantidades subinhibitorias al interior del biofilm e induce estrés a las bacterias provocando una expresión de los mecanismos de resistencia (30). 


\section{Alteración del microambiente}

En la parte interna del biofilm las concentraciones de oxígeno son demasiado bajas; debido a esta condición, las bacterias reducen sus actividades metabólicas y tiempo de replicación, lo cual produce una tolerancia a los antibióticos como los aminoglucosidos al bloquear sus rutas metabólicas (Figura 2.b) (29,30).

\section{Formación de células microbianas persistentes y de crecimiento lento}

Los biofilms contienen subpoblaciones bacterianas caracterizadas por una amplia variedad de actividad metabólica. Principalmente, aquellas bacterias que se encuentran en la periferia, presentan cambios morfológicos que impiden la acción de los antibióticos en su sitio diana (30), mientras que las que se encuentran ubicadas en la parte interna del biofilm no tienen crecimiento y tienen una baja actividad fisiológica. Esto se debe al consumo de oxígeno y nutrientes por parte las micro-colonias que se encuentran en la periferia (Figura 2.c) (31,32). Debido a su inactividad fisiológica, estas bacterias generan una baja síntesis de proteínas que afecta la eficiencia de antibióticos como el cloranfenicol y la tetraciclina, la baja síntesis de ADN afecta el efecto de las quinolinas y la baja síntesis de peptidoglicano afecta el efecto de los betalactámicos (33).

Elaboración propia con base: Peńa S. Biopelículas microbianas y su impacto en áreas médicas: fisiopatología, diagnóstico y tratamiento. 2018. Dentro de los factores que favorecen la tolerancia antimicrobiana, se encuentra la inhibición de la penetración en donde gracias a la matriz de EPS el antibiótico no logra penetrar el biofilm. También puede ocurrir la alteración del microambiente en donde -debido al bajo oxígeno en algunas partes del biofilm- las bacterias reducen su actividad metabólica. Por último, debido a la constante exposición a antibiótico se generan bacterias resistentes dentro del biofilm a algunos antibióticos.

\section{MECANISMOS DE RESISTENCIA MEDIADOS POR BIOFILM}

La efecto de los antibióticos ante enfermedades causadas por Pseudomonas aeruginosa puede verse afectada debido a una resistencia o tolerancia por parte de este microorganismo (34). La resistencia puede ocurrir de forma natural o puede ser causada por mutaciones genéticas debido a la constante exposición a antibióticos. Por otra parte, la
Figura 2. Esquema sobre los factores que favorecen del desarrollo de tolerancia antimicrobiana: a. inhibición de la penetración, b. alteración del microambiente, c. bacterias persistentes. Fuente: Autores.

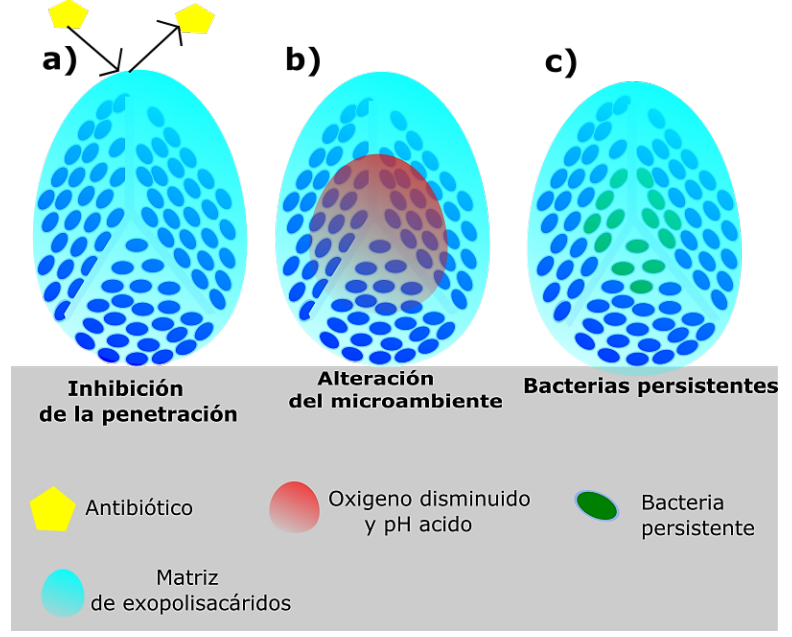

tolerancia se genera por una variación fenotípica transitoria que produce una supervivencia de estos microrganismos a concentraciones altas de antibióticos $(9,35)$.

Pseudomonas aeruginosa al crecer en estado de biofilm incrementa la tolerancia a los antibióticos hasta mil veces más que cuando crecen en su forma libre (36) y se asocia principalmente a los siguientes mecanismos: seńal de quorum sensing (Q.S), porinas, bombas de eflujo, expresión de genes, vesículas de membrana, $\mathrm{ADN}$ extracelular y enzimas.

\section{Quorum sensing}

El quorum sensing es un mecanismo de comunicación intercelular que permite a las bacterias dentro del biofilm adaptarse a los cambios microambientales. Esta dado por moléculas llamadas autoinductores, las cuales se difunden a través de la membrana bacteriana y activan receptores de membrana que desencadenan la expresión y regulación de los genes diana, que permiten localizar la densidad de las bacterias colonizantes $(21,37)$.

El mecanismo que las bacterias utilizan para comunicarse entre sí se produce a partir de la secreción y elaboración de "moléculas de seńalización", mediante las cuales se adaptan y coordinan de manera unificada. Están conformadas por tres sistemas: dos sistemas basados en acil homoserina lactona (el sistema Las y el sistema Rh1) y un sistema basado en quinolonas llamado en inglés Pseudomonas 
quinolone signal (PQS). Los Rh1 regulan la producción de ramnolípidos y el PQS regula la generación del ADN extracelular componente de la matriz, el GacA/GacS regulan la expresión de exopolisacáridos de Pel y Psl $(10,13)$.

Cuando los antibióticos logran penetrar la matriz de EPS y destruyen las matriz de la periferia, las subpoblaciones resistentes a este antibiótico migran a la parte superior del biofilm y emplean la seńal de quorum sensing para iniciar su replicación y de esta forma le concede tolerancia al biofilm sobre el antibiótico (9).

\section{Porinas}

La restricción del acceso de antibióticos mediado por porinas es uno de los mecanismos más comunes de la resistencia que actúa evitando el ingreso del antimicrobiano. Mediante esta proteína se puede disminuir la afinidad y transporte del antibiótico en cepas bacterianas que presenten mutaciones en la porina 0 prD. Estas porinas determinan la permeabilidad de la membrana externa por medio de canales que permiten la difusión de moléculas hidrofilicas y el transporte del agua; su modificación afecta la entrada del antibiótico a su sitio de acción. Dentro de las principales porinas que generan tolerancia al biofilm se encuentran la porina 0 prF y la porina 0 prD (38).

\section{Porina 0prD}

La porina OprD es una porina de membrana de $P$. aeruginosa y su función principal es la captación de aminoácidos a través de la membrana externa, además es capaz de permitir la entrada de carbapenémicos. La regulación negativa de OprD le proporciona una tolerancia fundamental contra carbapenémicos especialmente contra Imipenem $(39,40)$.

Esta regulación negativa está dada principalmente por los sistemas de transducción de seńales de dos componentes TCS, los sistemas reguladores CzcR-CzcS y CopR-CopS. Los TCS consisten principalmente en el sistema de transducción de seńales CzcR-CzcS.

El sistema CzcR es activado gracias a la unión de metales (zinc, cadmio, cobalto o cobre) al sensor de histidina quinasa que se encuentra en la membrana de la bacteria; la histidina se dimeriza y fosforila generando una activación de sistema CzcR, que posteriormente va a desencadenar un descenso de la transcripción de gen oprD lo cual genera una disminución de la expresión de la porina OprD en la membrana de los microorganismo dentro del biofilm $(39,41)$.

\section{Bombas de expulsión}

Los conjuntos de bombas de eflujo participan en la supervivencia del biofilm en ambientes extremos y frente a los antibióticos. Estas bombas de eflujo ejercen una tolerancia tanto intrínseca como adquirida a los diferentes antibióticos. Una sobreproducción de estas bombas ejercen un fenotipo de tolerancia combinado con la modificación de sitios diana y la inactivación de los antibióticos (29).

Las bombas de eflujo tienen la capacidad de bombear los antibióticos fuera de las bacterias por medio de un proceso que requiere energía y depende del potencial de membrana y ATP. Pseudomonas aeruginosa, puede inducir bombas cuando son expuestas al estrés. Dentro de ellas se encuentra la bomba mexXY-OprM inducida por estrés oxidativo que confiere resistencia a aminoglucosidos, MeEF.OprN inducina por estrés nitrosativo generando resistencia frente a fluoroquinolonas y MexcD-0prJ por dańo en la membrana que ocasiona resistencia contra los betalactámicos. Estos tipos de estrés en el biofilm inducen la producción de bombas de eflujo que contribuyen a la tolerancia del biofilm (21).

\section{Expresión de genes}

La adaptación genética es necesaria dentro del biofilm para reducir la susceptibilidad y adoptar el fenotipo para protegerse de los distintos cambios del ambiente y la exposición a los antibióticos $(42,43)$. P. aeruginosa en la región externa de un biofilm bloquea el funcionamiento de los antibióticos a través de la expresión de genes específicos que la protegen de los mismo; este es un tipo de respuesta adaptativa que se expresa constitutivamente e incluso antes de la exposición del antibiótico $(44,45)$.

La molécula mensajera c-di-GMP se encuentra elevada en las bacterias dentro del biofilm; cuando está en su forma planctónica genera una mayor tolerancia al biofilm. El regulador del locus de resistencia del biofilm BrlR es un activador transcripcional similar a Mer en la tolerancia específica del biofilm de P. aeruginosa. Además, es un activador transcripcional de las bombas de salida por los operones mexAB-orpM y mexEF-opr. La actividad del regulador transcripcional BrlR se estimula mediante la unión a la molécula c-di-GMP (46).

Uno de los genes más importantes en la tolerancia del biofilm es el gen ndvB ya que es el encargado de sintetizar 
la glucosiltrasnferasa NdvB que unida a la membrana produce los $\llbracket$ glucanos cíclicos los cuales se encuentran en el periplasma y la matriz extracelular de las biopelículas. Los glucanos cíclicos interactúan directamente con los aminoglucósidos (kanamicina, tobramicina) y evitan la ruptura de la membrana interna $(47,48)$.

\section{Vesículas de membrana}

Las vesículas que se encargan de transportar los nutrientes al interior del biofilm participan como un mecanismo de tolerancia. Su interior está conformado por proteínas que se unen a los fármacos los inactivan y los expulsan del biofilm. Además, también transportan betalactamasas que destruyen a algunos tipos de antibióticos.

Las vesículas que se encargan de transportar los nutrientes al interior del biofilm participan como un mecanismo de tolerancia. Su interior está conformado por proteínas que se unen a los fármacos los inactivan y los expulsan del biofilm. Además, también transportan betalactamasas que destruyen a algunos tipos de antibióticos.

\section{ADN extracelular}

El ADN extracelular cumple funciones importantes en el desarrollo y estabilidad de las biopelículas. Ayuda a la

Figura 3. Esquema que permite identificar los componentes que confieren tolerancia mediada por vesículas de membrana. Fuente: Autores.
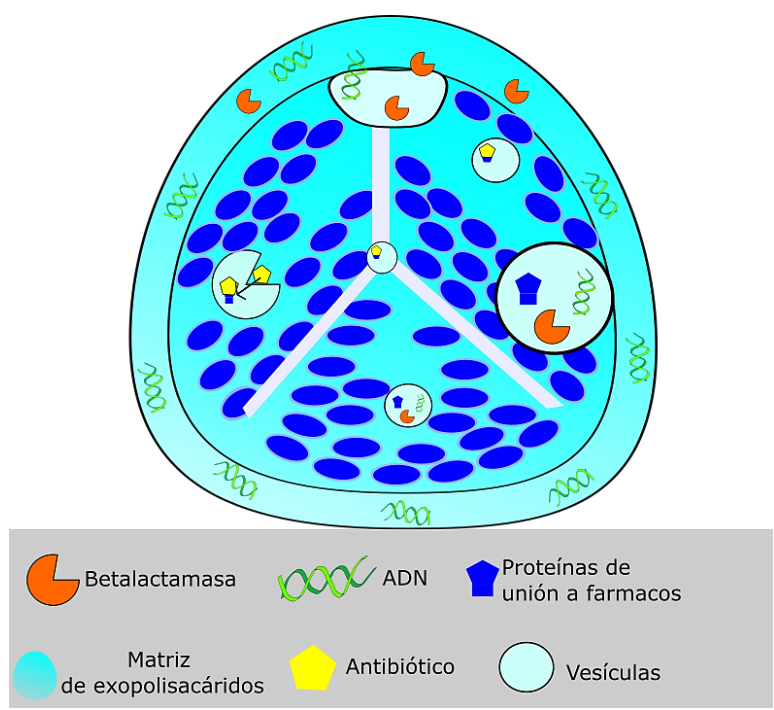

adhesión a la superficie modulando la interacción de carga e hidrofobicidad, además asegura la alineación adecuada dentro del biofilm lo cual facilita la expansión del mismo y proporciona una trasferencia horizontal de genes a las bacterias del biofilm (52). El ADN extracelular en la matriz del biofilm es un constituyente importante y mayoritario que va a permitir la conexión intercelular, convirtiéndose en un mecanismo de gran importancia en los procesos de resistencia a antibióticos por $P$. aeruginosa.

Los antibióticos cargados positivamente se unen al ADN cargado negativamente lo cual evita que el antibiótico haga efecto en su sitio de acción. Debido a que la matriz del biofilm puede saturarse por la unión del antibiótico al ADN, esta tolerancia es temporal mientras que permite que las bacterias expuestas puedan adaptarse y generar subpoblaciones tolerantes (10).

Otro mecanismo por el cual el ADN aumenta la resistencia del biofilm es gracias a su capacidad de quelar cationes como los iones de magnesio lo cual provoca una disminución del Mg en la membrana. Esto induce la activación de los componentes PhopQ y PmrAB lo cual proporciona una resistencia antimicrobiana ya que producen un reordenamiento del operon PA 33552-3559 el cual codifica una proteína que une la aminoarabinosa al lípido A (tolerancia contra el péptido catiónico) (Figura 4) (53).

Figura 4. Esquema de Tolerancia a antibióticos mediada por ADN. Fuente: Autores.

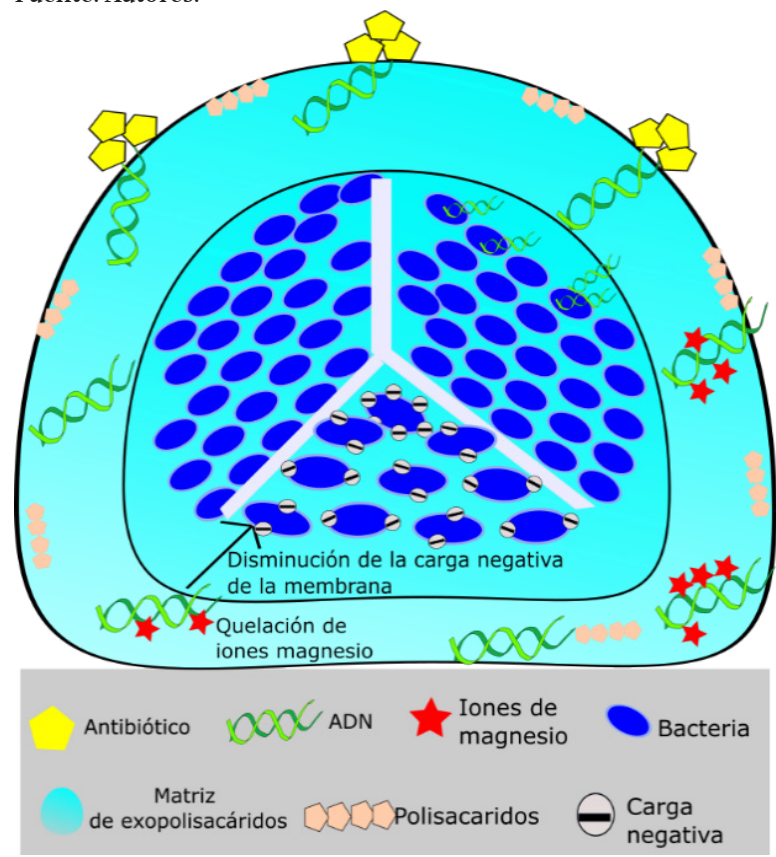


Figura 5. Esquema que permite identificar la Tolerancia a antibióticos mediada por enzimas. Fuente: Autores.

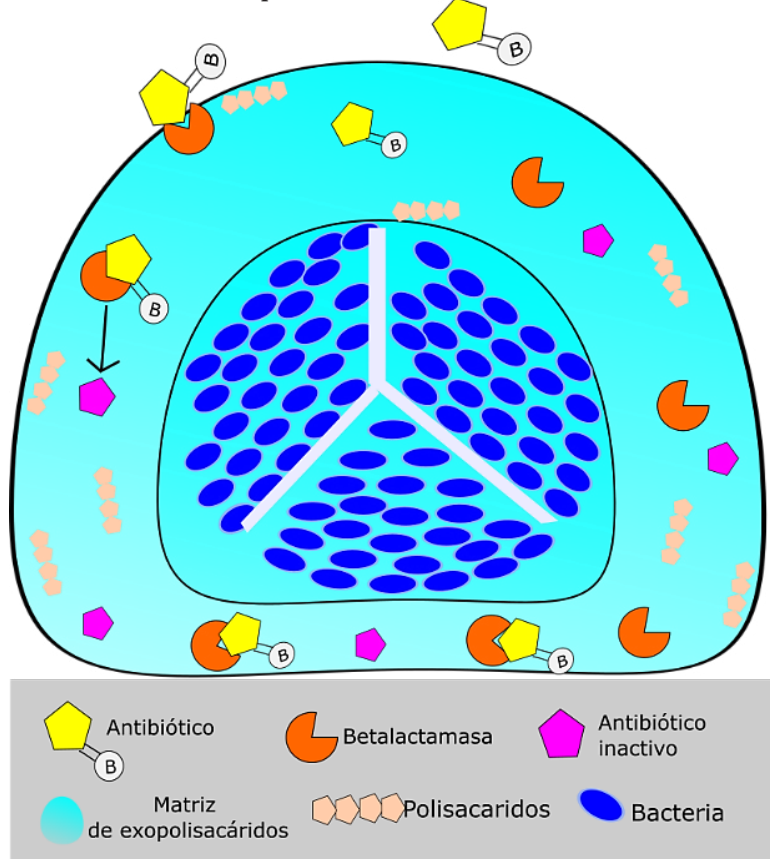

\section{TOLERANCIA MEDIADA POR ENZIMAS}

La tolerancia esta mediada por la trasformación del bactericida a una forma no toxica para el biofilm. Esto se logra gracias a la actividad de diferentes enzimas como la catalasa y betalactamasas, las cuales son recolectadas en grandes cantidades en la matriz de EPS. Por esta acumulación se aumenta la hidrolisis de antibióticos $(54,55)$.

\section{CONCLUSIONES}

La formación de biofilms de Pseudomonas aeruginosa se ha considerado como uno de los principales mecanismos de resistencia frente a antibióticos. Diversos mecanismos de resistencia que generan la destrucción o inhibición de la función de los antibióticos solo se expresan cuando se presenta la formación del biofilm y una vez que los microorganismos se liberan y vuelven a su forma planctónica, estos mecanismos ya no son expresados.

Uno de los mecanismos que ha cobrado interés a través del tiempo, es la expresión de genes ya que partir de estos es donde se inicia la traducción de los diferentes mecanismos de evasión. Desde la disminución de la penetración y disminución de la carga eléctrica mediados por ADN y la matriz de EPS, la destrucción de antibióticos gracias a las diferentes enzimas que se presentan en la matriz. Adicionalmente, dada la compleja arquitectura del biofilm, este genera diferentes factores microambientales que colaboran con la tolerancia a los diferentes tipos de antibióticos.

Finalmente, el reconocimiento e identificación de los diferentes biofilms como mecanismos de resistenciay tolerancia, abre el paso a la potencial generación de estrategias de prevención y tratamiento como una opción en la disminución de la transmisión de infecciones causadas por Pseudomonas aeruginosa.

\section{CONTRIBUCIÓN DE LOS AUTORES}

Andrés Felipe Bolívar Vargas, María Inés Torres Caycedo y Yaline Sánchez Neira: contribuyeron en la revisión de la redacción y escritura del manuscrito realizaron una contribución sustancial con la adquisición de los datos e información, así mismo para la escritura del artículo, su revisión y aprobación.

\section{CONFLICTOS DE INTERÉS}

Los autores declaran no tener ningún conflicto de interés en la realización de este escrito

\section{AGRADECIMIENTOS}

La Universidad de Boyacá, por su aporte en la formación académica y la disposición en procesos de investigación

\section{REFERENCIAS}

1. Dumaru R, Baral R, Shrestha LB. Study of biofilm formation and antibiotic resistance pattern of gramnegative Bacilli among the clinical isolates at BPKIHS, Dharan. BMC Res Notes. 2019; 12(1)

2. Villanueva-Ramos NB, De la Mora-Fernández AR, RíosBurgueńo ER, de Peraza-Garay FJ. Detección de biopelículas en tejido de amígdalas y adenoides en pacientes con procesos infecciosos crónicos y obstructivos. Anales de Otorrinolaringología Mexicana. 2019; 64 
3. Schiessl KT, Hu F, Jo J, Nazia SZ, Wang B, Price-Whelan A, et al. Phenazine production promotes antibiotic tolerance and metabolic heterogeneity in Pseudomonas aeruginosa biofilms. Nat Commun. 2019;10(1):1-10. Doi. https://doi. org/10.1038/s41467-019-08733-w

4. Romeo A. Biofilm y resistencia antimicrobiana. Arch Med camagüey. 2020; 24(4):1-4

5. Valen H, Scheie AA. Biofilms and their properties. Eur J Oral Sci. 2018; 126:13-8

6. Del Pozo JL. Biofilm-related disease. Expert Review of AntiInfective Therapy. Taylor and Francis Ltd. 2018; 16:51-65.

7. European Centre for Disease Prevention and Control, "Surveillance Report. Surveillance of antimicrobial resistance in Europe 2016". 2017

8. Kohlenberg, A.; Weitzel-Kage, D.; van der Linden, P. et al. Outbreak of Carbapenem-Resistant Pseudomonas aeruginosa Infection in a Surgical Intensive Care Unit. J. Hosp. Infect. 2010; 74(4):350-357

9. Naze, F.; Jouen, E.; Randriamahazo, R. T. et al. Pseudomonas aeruginosa Outbreak Linked to Mineral Water Bottles in a Neonatal Intensive Care Unit: Fast Typing by Use of High-Resolution Melting Analysis of a Variable-Number Tandem-Repeat Locus. J. Clin. Microbiol. 2010; 48(9):3146-3152

10. Chua SL, Yam JKH, Hao P, Adav SS, Salido MM, Liu Y, et al. Selective labelling and eradication of antibioticTolerant bacterial populations in Pseudomonas aeruginosa biofilms. Nat Commu. 2016; 7(1):1-11

11. Rybtke M, Hultqvist LD, Givskov M, Tolker-Nielsen T. Pseudomonas aeruginosa Biofilm Infections: Community Structure, Antimicrobial Tolerance and Immune Response. Journal of Molecular Biology. Academic Press. 2015; 427:3628-45

12. Peńa S-GG. Producción de biopelículas y resistencia antimicrobiana en uropatógenos aislados de catéteres urinarios en un hospital de rehabilitación física. 2017; 6(3):115-21

13. Heidari H, Hadadi M, Sedigh Ebrahim-Saraie H, Mirzaei A, Taji A, Hosseini SR, et al. Characterization of virulence factors, antimicrobial resistance patterns and biofilm formation of Pseudomonas aeruginosa and Staphylococcus spp. strains isolated from corneal infection. J Fr Ophtalmol. 2018; 41(9):823-9

14. Maurice NM, Bedi B, Sadikot RT. Pseudomonas aeruginosa biofilms: Host response and clinical implications in lung infections. Vol. 58, American Journal of Respiratory Cell and Molecular Biology. American Thoracic Society. 2018; 58:428-39
15. Rodrigues ME, Lopes SP, Pereira CR, Azevedo NF, Lourenço A, Henriques M, et al. Polymicrobial Ventilator-Associated Pneumonia: Fighting In Vitro Candida albicans-Pseudomonas aeruginosa Biofilms with Antifungal-Antibacterial Combination Therapy. Sturtevant J, editor. PLoS One. 2017; 12(1):e0170433

16. Zhou ZY, Hu BJ, Gao XD, Bao R, Chen M, Li HY. Sources of sporadic Pseudomonas aeruginosa colonizations/ infections in surgical ICUs: Association with contaminated sink trap. J Infect Chemother. 2016; 22(7):450-5

17. Pericolini E, Colombari B, Ferretti G, Iseppi R, Ardizzoni A, Girardis M, et al. Real-time monitoring of Pseudomonas aeruginosa biofilm formation on endotracheal tubes in vitro. BMC Microbiol. 2018;18(1)

18. Gil-Perotin S, Ramirez P, Marti V, Sahuquillo JM, Gonzalez E, Calleja I, et al. Implications of endotracheal tube biofilm in ventilator-associated pneumonia response: A state of concept. Crit Care. 2012; 16(3):R93

19. Werneburg GT, Nguyen A, Henderson NS, Rackley RR, Shoskes DA, Le Sueur AL, et al. The Natural History and Composition of Urinary Catheter Biofilms: Early Uropathogen Colonization with Intraluminal and Distal Predominance. J Urol. 2020; 203(2):357-64

20. Stickler DJ. Clinical complications of urinary catheters caused by crystalline biofilms: something needs to be done. J Intern Med. 2014; 276(2):120-9. Doi: http://doi. wiley.com/10.1111/joim. 12220

21. Oluyombo 0, Penfold CN, Diggle SP. Competition in biofilms between cystic fibrosis isolates of Pseudomonas aeruginosa is shaped by R-pyocins. MBio. 2019;10(1). Doi: https:// doi.org/10.1128/mBio.01828-18

22. Zarza VMP, Mordani SM, Maldonado AM, Hernández DÁ, Georgina SGS, Vázquez-López R. Pseudomonas aeruginosa: Pathogenicity and antimicrobial resistance in urinary tract infection. Rev Chil Infectol. 2019; 36(2):180-9

23. Jerković-Mujkić A. Biofilm formation and antimicrobial susceptibility of Pseudomonas aeruginosa in the indoor and outdoor environment (CIBTech Journal of Microbiology). J Microbiol. 2019; 8(2):23-30

24. Thanabalasuriar A, Scott BNV, Peiseler M, Willson ME, Zeng Z, Warrener P, et al. Neutrophil Extracellular Traps Confine Pseudomonas aeruginosa Ocular Biofilms and Restrict Brain Invasion. Cell Host Microbe. 2019; 25(4):526-536.e4

25. Olivares E, Badel-Berchoux S, Provot C, Prévost G, Bernardi T, Jehl F. Clinical Impact of Antibiotics for the Treatment of Pseudomonas aeruginosa Biofilm Infections. Front Microbiol. 2020; 10(2):12-32 
26. Rasamiravaka T, Labtani Q, Duez P, El Jaziri M. The formation of biofilms by Pseudomonas aeruginosa: A review of the natural and synthetic compounds interfering with control mechanisms. Biomed Res Int. 2015;2015(7):17

27. Bermúdez, Lázaro-Gonzáles M. La biopelícula: una nueva concepción de la placa dentobacteriana. mediocentro Electron. 2016; 20(3):2-10

28. Arciola CR, Campoccia D, Montanaro L. Implant infections: Adhesion, biofilm formation and immune evasion. Nature Reviews Microbiology. Nature Publishing Group. 2018; 16:397-409

29. Xu X, Yu H, Zhang D, Xiong J, Qiu J, Xin R, et al. Role of ppGpp in Pseudomonas aeruginosa acute pulmonary infection and virulence regulation. Microbiological Research 2016;192:84-95. https://doi.org/10.1016/j. micres.2016.06.005.

30. Singh S, Singh SK, Chowdhury I, Singh R. Understanding the Mechanism of Bacterial Biofilms Resistance to Antimicrobial Agents. Open Microbiol J. 2017; 11(1):53-62

31. Ortega-Peńa S, Hernández-Zamora E. Biopelículas microbianas y su impacto en áreas médicas: fisiopatología, diagnóstico y tratamiento. 2018; 75:79-88

32. Mulet Aguiló FJ. Caracterización del mutante nfxB de Pseudomonas aeruginosa: papel en la resistencia antibiótica de los biofilms e interacción con los mecanismos de resistencia intrínsecos. Universitat de les Illes Balears; 2015;7

33. Stewart PS, Zhang T, Xu R, Pitts B, Walters MC, Roe F, et al. Reaction-diffusion theory explains hypoxia and heterogeneous growth within microbial biofilms associated with chronic infections. npj Biofilms Microbiomes. 2016; 2:16012

34. Yan J, Bassler BL. Surviving as a Community: Antibiotic Tolerance and Persistence in Bacterial Biofilms. Cell Host and Microbe. Cell Press. 2019; 26:15-21

35. Dumaru R, Baral R, Shrestha LB. Study of biofilm formation and antibiotic resistance pattern of gramnegative Bacilli among the clinical isolates at BPKIHS, Dharan. BMC Res Notes. 2019; 12(1):38

36. Azam MW, Khan AU. Updates on the pathogenicity status of Pseudomonas aeruginosa. Drug Discovery Today. Elsevier Ltd. 2019; 24:350-9

37. Stewart PS. Antimicrobial Tolerance in Biofilms. Microbiol Spectr. 2015; 3(3):16-10

38. Brindhadevi K, LewisOscar F, Mylonakis E, Shanmugam S, Verma TN, Pugazhendhi A. Biofilm and Quorum sensing mediated pathogenicity in Pseudomonas aeruginosa. Process Biochemistry. Elsevier Ltd. 2020; 96:49-57
39. Troncoso C, Pavez M, Santos A, Salazar R, Barrientos Díaz L. Implicancias estructurales y fisiológicas de la célula bacteriana en los mecanismos de resistencia antibiótica. Int J Morphol. 2017; 35(4):1214-23

40. Raavi, Mishra S, Singh S. Prevention of OprD regulated antibiotic resistance in Pseudomonas aeruginosa biofilm. Microb Pathog. 2017; 112:221-9

41. Al Marjania MF, Kouhsari E, Ali FS, Authman SH. Evaluation of type II toxin-antitoxin systems, antibiotic resistance profiles, and biofilm quorum sensing genes in Acinetobacter baumannii isolates in Iraq. Infect Disord - Drug Targets. 2020; 20:133-9

42. Chevalier S, Bouffartigues E, Bodilis J, Maillot 0 , Lesouhaitier 0, Feuilloley MGJ, et al. Structure, function and regulation of Pseudomonas aeruginosa porins. FEMS Microbiology Reviews. Oxford University Press; 2017; 41:698-722

43. Stewart PS, Franklin MJ, Williamson KS, Folsom JP, Boegli L, James GA. Contribution of stress responses to antibiotic tolerance in Pseudomonas aeruginosa biofilms. Antimicrob Agents Chemother. 2015; 59(7):3838-47

44. RochaAJ,DeOliveiraBarsottini MR, RochaRR, Laurindo MV, De Moraes FLL, Da Rocha SL. Pseudomonas aeruginosa: Virulence factors and antibiotic resistance Genes. Vol. 62, Brazilian Archives of Biology and Technology. Instituto de Tecnologia do Parana. 2019; 62:1-15

45. Karami P, Khaledi A, Mashoof RY, Yaghoobi MH, Karami M, Dastan D, et al. The correlation between biofilm formation capability and antibiotic resistance pattern in Pseudomonas aeruginosa. Gene Reports. 2020; 18:100561

46. Ghazalibina M, Morshedi K, Farahani RK, Babadi M, Khaledi A. Study of virulence genes and related with biofilm formation in Pseudomonas aeruginosa isolated from clinical samples of Iranian patients; A systematic review. Gene Reports. Elsevier Inc. 2019; $17: 100471$

47. Ciofu 0, Tolker-Nielsen T. Tolerance and resistance of Pseudomonas aeruginosa biofilms to antimicrobial agents-how P. aeruginosaCan escape antibiotics. Frontiers in Microbiology. Frontiers Media S.A. 2019; 10:913

48. Hall CW, Mah TF. Molecular mechanisms of biofilmbased antibiotic resistance and tolerance in pathogenic bacteria. FEMS Microbiology Reviews. Oxford University Press. 2017; 41:276-301

49. Miryala SK, Anbarasu A, Ramaiah S. Systems biology studies in Pseudomonas aeruginosa PA01 to understand their role in biofilm formation and multidrug efflux pumps. Microb Pathog. 2019;136 
50. Wang W, Chanda W, Zhong M. The relationship between biofilm and outer membrane vesicles: A novel therapy overview. FEMS Microbiol Lett. 2015; 362(15):117

51. Cooke AC, Nello A V., Ernst RK, Schertzer JW. Analysis of Pseudomonas aeruginosa biofilm membrane vesicles supports multiple mechanisms of biogenesis. Hozbor DF, editor. PLoS One. 2019; 14(2):e0212275

52. Vitse J, Devreese B. The Contribution of Membrane Vesicles to Bacterial Pathogenicity in Cystic Fibrosis Infections and Healthcare Associated Pneumonia. Front Microbio. 2020; 11:630

53. Wilton M, Charron-Mazenod L, Moore R, Lewenza S. Extracellular DNA acidifies biofilms and induces aminoglycoside resistance in Pseudomonas aeruginosa. Antimicrob Agents Chemother. 2016; 60(1):544-53

54. Li Y, Xiao P, Wang Y, Hao Y. Mechanisms and control measures of mature biofilm resistance to antimicrobial agents in the clinical context. ACS Omega. American Chemical Society. 2020; 5:22684-90. Doi: https:// dx.doi.org/10.1021/acsomega.0c02294

55. Al-Wrafy F, Brzozowska E, Górska S, Gamian A. Pathogenic factors of Pseudomonas aeruginosa - the role of biofilm in pathogenicity and as a target for phage therapy. Postepy Higieny i Medycyny Doswiadczalnej. Polska Akademia Nauk. 2017; 71:78-91 